\title{
Semi-Isolation and the Strict Order Property
}

\author{
Sergey Sudoplatov and Predrag Tanović
}

\begin{abstract}
We study semi-isolation as a binary relation on the locus of a complete type and prove that—under some additional assumptions—it induces the strict order property.
\end{abstract}

\section{Introduction}

Throughout the paper $T$ is a fixed, complete, first-order theory in a countable language and $M$ is its (infinite) monster model. $T$ is an Ehrenfeucht theory if it has finitely many, but more than one, countable models. The class of Ehrenfeucht theories is quite interesting. There are numerous results and a large bibliography in this area (see Baizhanov, Sudoplatov, and Verbovskiy [1] and Sudoplatov [8] for references). The first example was found by Ehrenfeucht in Vaught [11, Section 6]: $T_{E}=\operatorname{Th}(\mathbb{Q},<, n)_{n \in \omega}$. It eliminates quantifiers and has three countable models: the prime model, the saturated model, and the model prime over a realization of a nonisolated type. $T_{E}$ is also a binary theory: every formula is equivalent modulo $T_{E}$ to a Boolean combination of formulas with at most two free variables. Not all Ehrenfeucht theories are binary: nonbinary examples can be found in Peretyat'kin [4] and Woodrow [13]. The motivating question for our work is the following.

Question 1 Is there a binary, Ehrenfeucht theory without the strict order property (SOP)? In particular, is there such a theory with three countable models?

An important relation in any Ehrenfeucht theory is semi-isolation as a binary relation on the locus of a powerful type $p \in S(\emptyset)$ in a model of $T$ (all these notions are defined in Section 1). There the semi-isolation relation is either empty (if $p$ is omitted) or a $\bigvee$-definable quasiorder with no maximal elements. If in addition $T$ has precisely three countable models, then the isomorphism type of any countable model $N$ can be described by combinatorial properties of the quasiorder:

Received November 12, 2012; accepted August 27, 2013

2010 Mathematics Subject Classification: Primary 03C15; Secondary 03C45

Keywords: small theory, nonisolated type, semi-isolation, powerful type

(C) 2015 by University of Notre Dame 10.1215/00294527-3153579 
1. $N$ is prime if and only if $p(N)=\emptyset$;

2. $N$ is prime over a realization of $p$ if and only if there is a minimal (with respect to semi-isolation) element in $p(N)$; in this case, $N$ is prime over any minimal element;

3. $N$ is saturated if and only if $p(N) \neq \varnothing$ has no minimal elements.

We note that in Ehrenfeucht's example the type $\{n<x \mid n \in \omega\}$ determines a complete 1-type $p$ on whose locus, in any countable model, the semi-isolation (defined precisely later and denoted by $\mathrm{SI}_{p}$ ) coincides with $\leq$. In particular, semiisolation is a relatively definable relation on the locus of $p$. The strict order property in this example is induced by the semi-isolation, and it is natural to examine whether this will happen in any binary Ehrenfeucht theory.

One result in this direction was obtained by Woodrow in [12]. He proved that if a theory in the language of Ehrenfeucht's example eliminates quantifiers and has three countable models, then it is quite similar to the original one; in particular, semiisolation is a relatively definable ordering on the locus of a powerful type. Ikeda, Pillay, and Tsuboi proved that the same happens in the case of an almost $\boldsymbol{\aleph}_{0}$-categorical theory with three countable models (see [3, Theorem 7]). Another result in this direction was obtained by Pillay in [5, Theorem 5], who proved that in any Ehrenfeucht theory with few links there exists a definable linear ordering. The ordering relation that he found, when restricted to the locus of a powerful type, is induced by the semi-isolation relation.

In this article we will investigate proper quasiorders of the form $\left(p(M), \mathrm{SI}_{p}\right)$, where $p \in S(\emptyset)$ is a nonisolated type in an arbitrary first-order theory, and prove that, under some additional assumptions, a relatively definable suborder can be found. The additional assumptions have a topological flavor. That is not surprising because $\mathrm{SI}_{p}$ has a natural topological "definition" as a subspace of the compact space $S_{p, p}$ consisting of all complete extensions of $p(x) \cup p(y)$. The semi-isolation $\mathrm{SI}_{p}$ corresponds to the subspace $S_{\rightarrow}^{p}$ of all types $\operatorname{tp}(a, b)$, where $(a, b) \in \mathrm{SI}_{p}$. We will decompose $S_{p, p}$ into four parts, adequate for studying definability properties of $\mathrm{SI}_{p}$ (see Definition 1.1 and Remark 1.2). Then we will translate definability properties of semi-isolation into topological (complexity) properties of these parts.

In Section 2 we will prove that certain assumptions on the complexity imply the existence of a proper, relatively definable suborder of $\mathrm{SI}_{p}$. For example, we will prove in Theorem 2.7 that if the theory $T$ has closed asymmetric links on $p(M)$ (meaning that one of the parts, the set $S_{\mapsto}^{p}$, is nonempty and closed in $S_{p, p}$ ), then there exists a nontrivial, relatively definable suborder of $\mathrm{SI}_{p}$. This is one direction in which we generalize Pillay's result: if $p$ is a powerful type of an Ehrenfeucht theory with few links, then $S_{\mapsto}^{p}$ is finite (hence closed) and nonempty.

In Sections 3 and 4 we concentrate on the existence of antichains in $\mathrm{SI}_{p}$ in the case of the negation of the strict order property (NSOP), that is the case in which there is no formula $\varphi(\bar{x}, \bar{y})$ of given theory and tuples $\bar{a}_{i}, i \in \omega$, such that the following equivalence holds:

$$
\vdash \varphi\left(\bar{a}_{i}, \bar{y}\right) \rightarrow \varphi\left(\bar{a}_{j}, \bar{y}\right) \Leftrightarrow i \leq j .
$$

We do not do much in this direction: assuming that the underlying theory is binary, NSOP, and has three countable models, with lots of effort we prove that there are at least two distinct types of SI $p$-incomparable pairs of elements on the locus of a powerful type. This indicates that the answer to Question 1 may be affirmative. 
In Section 5 we consider a powerful type $p$ in a binary theory for which $\mathrm{SI}_{p}$ is downwards directed in a specific way (PGPIP; see Definition 5.1). We prove that in the NSOP case the Cantor-Bendixson rank of $S_{p, p}$ is finite, indicating that maybe there is no binary, Ehrenfeucht, NSOP theory with PGPIP at all. So the answer to Question 1 may be negative after all.

\section{Preliminaries}

Throughout the paper $S_{n}(A)$ denotes the set of all complete $n$-types with parameters from $A$. The topology on $S_{n}(A)$ is defined in the usual way. If $\varphi(\bar{x})$ is a formula over $A$ in $n$ free variables, then by $[\varphi]$ we will denote the set of all types from $S_{n}(A)$ containing $\varphi(\bar{x})$. The set $S(A)$ denotes $\bigcup_{n} S_{n}(A)$. If $p, q \in S(\emptyset)$, then $S_{p, q}(\varnothing)$ is the subspace of all the extensions of $p(\bar{x}) \cup q(\bar{y})$ in $S_{m}(\emptyset)$ (where $\bar{x}$ and $\bar{y}$ are disjoint and $m=|\bar{x}|+|\bar{y}|)$. Similarly, if $q \in S_{n}(\emptyset)$, then $S_{q}(A)$ denotes the set of all completions of $q(\bar{x})$ in $S_{n}(A)$. For any $\bar{c}$ realizing $p$ there is a canonical homeomorphism between $S_{p, q}(\emptyset)$ and $S_{q}(\bar{c})$ : the one sending $r(\bar{x}, \bar{y})$ to $r(\bar{c}, \bar{y})$.

Next we recall the definition of the Cantor-Bendixson rank. It is defined on the elements of a topological space $X$ by induction: $\mathrm{CB}_{X}(p) \geq 0$ for all $p \in X$; $\mathrm{CB}_{X}(p) \geq \alpha$ if and only if for any $\beta<\alpha, p$ is an accumulation point of the points of $\mathrm{CB}_{X}$-rank at least $\beta$. We have that $\mathrm{CB}_{X}(p)=\alpha$ if and only if both $\mathrm{CB}_{X}(p) \geq \alpha$ and $\mathrm{CB}_{X}(p) \nsucceq \alpha+1$ hold; if such an ordinal $\alpha$ does not exist, then $\mathrm{CB}_{X}(p)=\infty$. Isolated points of $X$ are precisely those having rank 0; points of rank 1 are those which are isolated in the subspace of all nonisolated points. For a nonempty $C \subseteq X$ we define $\mathrm{CB}_{X}(C)=\sup \left\{\mathrm{CB}_{X}(p) \mid p \in C\right\}$; in this way $\mathrm{CB}_{X}(X)$ is defined and $\mathrm{CB}_{X}(\{p\})=\mathrm{CB}_{X}(p)$ holds. If $X$ is compact and Hausdorff and $C$ is closed in $X$, then the sup is achieved: $\mathrm{CB}_{X}(C)$ is the maximum value of $\mathrm{CB}_{X}(p)$ for $p \in C$; there are finitely many points of maximum rank in $C$, and the number of such points is the $\mathrm{CB}_{X}$-degree of $C$. If $X$ is countable and compact, then $\mathrm{CB}_{X}(X)$ is a countable ordinal and every closed subset has ordinal-valued rank and finite $\mathrm{CB}_{X}$-degree.

$S_{n}(A)$ is compact, so CB-rank is defined there on points (complete types) and is well behaved on closed subsets (they correspond to partial types). So whenever $p$ is a partial type in $n$ free variables and parameters from $A$, then $\mathrm{CB}_{n}^{A}(p)$ is the CB-rank of the compact space consisting of all completions of $p$ in $S_{n}(A)$; usually the meaning of $n$ and $A$ will be clear from the context, so we will simply write $\mathrm{CB}(p)$. Similarly, the CB-degree is defined. Thus the CB-rank and degree are defined on all partial types and, in particular, they are defined on formulas. If $T$ is small (i.e., $\left.|S(\emptyset)|=\aleph_{0}\right)$, then the CB-rank of any partial type over a finite domain is an ordinal.

$\varphi(M, \bar{a})$ denotes the solution set of $\varphi(\bar{x}, \bar{a})$; if $p(\bar{x})$ is a (partial) type, then by $p(M)$ we denote the set of all its realizations. $D \subseteq M^{n}$ is definable if it is defined by a formula with parameters; it is $A$-definable (or definable over $A$ ) if the defining formula can be chosen to use only parameters from $A$. We have that $D$ is typedefinable ( $\bigvee$-definable) if it is the intersection (union) of $<|M|$ definable sets; if all the sets in the intersection (union) are definable over a fixed set $A \subset M$, then we say that $D$ is type-definable ( $\bigvee$-definable) over $A$. In this paper we will consider only countable intersections and unions of sets definable over a finite parameter set. Let $C \subseteq M^{n}$ be type-definable, and let $C_{1} \subseteq C$. Then $C_{1}$ is relatively definable within $C$ if there is a definable $D \subseteq M$ such that $C_{1}=C \cap D$; similarly, relative $\bigvee$-definability is defined. 
Semi-isolation was introduced by Pillay in [5]; here we will sketch its basic properties (the reader may find more details in [1]). $\bar{b}$ is semi-isolated over $\bar{a}$ (or $\bar{a}$ semi-isolates $\bar{b})$ if and only if there is a formula $\varphi(\bar{a}, \bar{x}) \in \operatorname{tp}(\bar{b} / \bar{a})$ such that $\varphi(\bar{a}, \bar{x}) \vdash \operatorname{tp}(\bar{b})$; we will denote that by $\bar{b} \in \operatorname{Sem}(\bar{a})$ or by $\bar{a} \rightarrow \bar{b} . \varphi(\bar{x}, \bar{y})$ is said to witness the semi-isolation; we will also write $\bar{a} \stackrel{\varphi}{\rightarrow} \bar{b}(\bar{a} \varphi$-arrows $\bar{b})$. Thus

$$
\bar{a} \stackrel{\varphi}{\rightarrow} \bar{b} \quad \text { if and only if } \models \varphi(\bar{a}, \bar{b}) \text { and } \varphi(\bar{a}, \bar{y}) \vdash \operatorname{tp}_{\bar{y}}(\bar{b}) \text {. }
$$

If $\bar{a} \rightarrow \bar{b}$, then there are many formulas witnessing the semi-isolation: if $\varphi(\bar{x}, \bar{y})$ is a witness, then $\varphi(\bar{x}, \bar{y}) \wedge \bar{x}=\bar{x}$ is a witness too. Therefore we can have many distinct named arrows between a fixed pair of tuples.

The reader may note that our definition of $\bar{a} \rightarrow \bar{b}$ does not exclude the existence of an arrow in the opposite direction. If, in addition to $\bar{a} \rightarrow \bar{b}$, we know that the opposite arrow does not exist (i.e., that $a \notin \operatorname{Sem}(b)$ ), we will write $\bar{a} \mapsto \bar{b}$. Therefore $\bar{a} \mapsto \bar{b}$ means that both $\bar{a} \rightarrow \bar{b}$ and $\bar{a} \notin \operatorname{Sem}(\bar{b})$ hold; $\bar{a} \rightarrow \bar{b}$ and $\bar{a} \mapsto \bar{b}$ may be consistent. $\bar{a} \leftrightarrow \bar{b}$ means $\bar{b} \mapsto \bar{a}$. And $a \stackrel{\varphi}{\mapsto} b$ means that both $a \stackrel{\varphi}{\rightarrow} b$ and $a \mapsto b$ hold, while $\bar{a} \leftrightarrow \bar{b}$ means that both $\bar{a} \rightarrow \bar{b}$ and $\bar{b} \rightarrow \bar{a}$ hold.

Consider semi-isolation as a binary relation on $M^{<\omega}$. It is trivially reflexive and it is not hard to see that it is transitive:

$$
\bar{a} \stackrel{\varphi}{\rightarrow} \bar{b} \text { and } \bar{b} \stackrel{\psi}{\rightarrow} \bar{c} \text { together imply } \bar{a} \stackrel{\varphi}{\rightarrow} \bar{c},
$$

where $\varphi(\bar{x}, \bar{z})$ is $\exists \bar{y}(\varphi(\bar{x}, \bar{y}) \wedge \psi(\bar{y}, \bar{z}))$. Thus semi-isolation is a quasiorder on $M^{<\omega}$. We note an interesting consequence of transitivity:

$$
\bar{a} \mapsto \bar{b} \rightarrow \bar{c} \text { implies } \bar{a} \mapsto \bar{c} \text {. }
$$

We will be interested mainly in semi-isolation as a binary relation on the locus of a complete type $p \in S(\emptyset)$. Then it is relatively $\bigvee$-definable within the locus: to simplify notation we will consider only 1-types; this is justified by passing to an appropriate sort in $M^{e q}$. So fix for a while $p \in S_{1}(\emptyset)$. Define

$$
\mathrm{SI}_{p}=\left\{(a, b) \in p(M)^{2} \mid a \rightarrow b\right\} .
$$

For any $(a, b) \in \mathrm{SI}_{p}$ there exists an $L$-formula $\varphi(x, y)$ witnessing $p$-semi-isolation. This implies that $\mathrm{SI}_{p}$ is defined by $\bigvee \varphi(x, y)$ within $p(M)^{2}$ (here the disjunction is taken over all such $\varphi$ 's), so $\mathrm{SI}_{p}$ is a relatively $\bigvee$-definable subset of $p(M)^{2}$.

Define

$$
\overline{\mathrm{SI}}_{p}=\left\{(a, b) \in p(M)^{2} \mid a \rightarrow b \text { or } b \rightarrow a \text { holds }\right\}, \quad \perp_{p}=p(M)^{2} \backslash \overline{\mathrm{SI}}_{p} .
$$

$(a, b) \in \perp_{p}$ means that $a, b$ are incomparable in the quasiorder, in which case we will write $a \perp_{p} b$. The semi-isolation $\overline{\mathrm{SI}}_{p}$ is relatively $\bigvee$-definable within $p(M)^{2}$, while $\perp_{p}$ is type-definable.

We will use the following syntax: $x \notin \operatorname{Sem}_{p}(y)$ will denote the type consisting of all negated formulas witnessing that $y p$-semi-isolates $x ; x \perp^{p} y$ will denote the type $x \notin \operatorname{Sem}_{p}(y) \cup y \notin \operatorname{Sem}_{p}(x)$. Therefore the type $p(x) \cup p(y) \cup x \perp^{p} y$ defines the set $\left\{(a, b) \in p(M)^{2} \mid a \perp_{p} b\right\}$ whose complement in $p(M)^{2}$ is $\overline{\mathrm{SI}}_{p}$.

Each $\varphi(x, y)$ witnessing $p$-semi-isolation defines a binary relation on $p(M)$, so the quasiorder $\mathrm{SI}_{p}$ may also be viewed as the union of a family of binary relations; this has already been suggested by the arrows notation. The relations defined by arrows correspond naturally to subsets of $S_{p, p}$, and relative definability properties translate into topological properties of these subsets. 
Definition 1.1 For a nonisolated $p \in S(\emptyset)$ and $\sigma \in\{\mapsto, \leftrightarrow, \rightarrow, \leftarrow, \leftrightarrow, \perp\}$, define

$$
S_{\sigma}^{p}=\left\{\operatorname{tp}(a b) \in S_{p, p} \mid a \sigma b\right\} .
$$

The nonisolation of $p$ in the definition is assumed in order to exclude the trivial case $\mathrm{SI}_{p}=p(M)^{2}$, which is not interesting at all.

Remark 1.2 Let $p \in S(\emptyset)$ be nonisolated. We list some observations related to the defined parts of $S_{p, p}$.

(1) $S_{\mapsto}^{p} \cup S_{\leftrightarrow}^{p}=S_{\rightarrow}^{p}$ and $S_{\leftarrow}^{p} \cup S_{\leftrightarrow}^{p}=S_{\leftarrow}^{p}$. We have that $S_{p, p}$ is the disjoint union

$$
S_{p, p}=S_{\mapsto}^{p} \dot{U} S_{\leftrightarrow}^{p} \dot{\cup} S_{\perp}^{p} \dot{U} S_{\leftrightarrow}^{p} .
$$

(2) The mapping taking $\operatorname{tp}(a, b)$ to $\operatorname{tp}(b, a)$ is a homeomorphism of $S_{p, p}$. It fixes setwise $S_{\perp}^{p}$ and $S_{\leftrightarrow}^{p}$ and maps $S_{\mapsto}^{p}$ onto $S_{\leftarrow}^{p}$ and $S_{\rightarrow}^{p}$ onto $S_{\leftarrow}^{p}$. In particular, $S_{\mapsto}^{p}$ and $S_{\leftarrow}^{p}$, as well as $S_{\rightarrow}^{p}$ and $S_{\leftarrow}^{p}$ are homeomorphic.

(3) $S_{\leftrightarrow}^{p}$ has at least one member (containing $x=y$ ). We have that $S_{\leftrightarrow}^{p} \neq S_{p, p}$ holds; otherwise, there would be a formula $\varphi(x, y)$ witnessing that each of $x$ and $y p$-semi-isolates the other such that $p(x) \cup p(y) \vdash \varphi(x, y)$. Then, by compactness, there would be $\theta(x) \in p$ such that $\models(\theta(x) \wedge \theta(y)) \Rightarrow \varphi(x, y)$ and, if $a \models p$ and $b \in \theta(M) \backslash p(M)$, we would get $\models \varphi(a, b)$, which is not possible by our choice of $\varphi(x, y)$.

(4) Each of $S_{\mapsto}^{p}, S_{\leftrightarrow}^{p}$, and $S_{\perp}^{p}$ may be empty while their union is nonempty (because of $\left.S_{\leftrightarrow}^{p} \neq S_{p, p}\right)$. By part (2), $S_{\mapsto}^{p}$ and $S_{\leftrightarrow}^{p}$ are homeomorphic, so they are either both empty or both nonempty.

- Consider the theory of an infinite set with infinitely many elements named, and let $p \in S_{1}(\emptyset)$ be the unique nonalgebraic type. Then $S_{\mapsto}^{p}=S_{\leftarrow}^{p}=\varnothing$, while $S_{\perp}^{p}$ is a singleton with a member containing $x \neq y$.

- Consider the type $p \in S_{1}(\emptyset)$ containing $\{n<x \mid n \in \omega\}$ in Ehrenfeucht's theory $T_{E}$. There $S_{\mapsto}^{p}$ and $S_{\leftarrow}^{p}$ have members containing $x<y$ and $y<x$, respectively, while $S_{\perp}^{p}=\emptyset$ because any two elements are comparable.

(5) $S_{\rightarrow}^{p}, S_{\leftarrow}^{p}$, and $S_{\leftrightarrow}^{p}$ are open in $S_{p, p}: S_{\rightarrow}^{p}$ is open because $S_{\rightarrow}^{p}=\bigcup_{\varphi}[\varphi]$, where the union is taken over all formulas $\varphi(x, y)$ witnessing $p$-semi-isolation; by homeomorphism, $S_{\leftarrow}^{p}$ is open too. If $\operatorname{tp}(a, b) \in S_{\leftrightarrow}^{p}$, then there is a formula $\varphi(x, y) \in \operatorname{tp}(a, b)$ witnessing $a \leftrightarrow b$ and $S_{\leftrightarrow}^{p}$ is the union $\bigcup_{\varphi}[\varphi]$ taken over all such $\varphi(x, y)$. And so $S_{\leftrightarrow}^{p}$ is open in $S_{p, p}$.

(6) $S_{\perp}^{p}$ is closed in $S_{p, p}$ because it is the set of all completions of $p(x) \cup p(y) \cup$ $x \perp^{p} y$.

(7) Since $\mathrm{SI}_{p}$ corresponds to $S_{\rightarrow}^{p}, \mathrm{SI}_{p}$ is relatively definable within $p(M)^{2}$ if and only if $S_{\rightarrow}^{p}$ is clopen in $S_{p, p}$. But $S_{\rightarrow}^{p}$ is always open, so $\mathrm{SI}_{p}$ is relatively definable if and only if $S_{\rightarrow}^{p}$ is closed in $S_{p, p}$.

(8) $\overline{\mathrm{SI}}_{p}$ corresponds to $S_{\rightarrow}^{p} \cup S_{\leftarrow}^{p}$, which is open. Therefore relative definability of $\overline{\mathrm{SI}}_{p}$ within $p(M)^{2}$ is equivalent to any of the following conditions:

- $S_{\rightarrow}^{p} \cup S_{\leftarrow}^{p}$ is clopen in $S_{p, p}$;

- $S_{\rightarrow}^{p} \cup S_{\leftarrow}^{p}$ is closed in $S_{p, p}$;

- $S_{\perp}^{p}$ is clopen in $S_{p, p}$ (because it is the relative complement of $S_{\rightarrow}^{p} \cup S_{\leftarrow}^{p}$ ). 
(9) We have $\operatorname{cl}\left(S_{\mapsto}^{p}\right) \subseteq S_{\mapsto}^{p} \cup S_{\perp}^{p}$ (where cl denotes the topological closure in $\left.S_{p, p}\right)$. Since $S_{\leftarrow}^{p}$ is open and disjoint from $S_{\mapsto}^{p}$, we have $\operatorname{cl}\left(S_{\mapsto}^{p}\right) \subseteq S_{p, p} \backslash$ $S_{\leftarrow}^{p}=S_{\mapsto}^{p} \cup S_{\perp}^{p}$. In particular, if $S_{\mapsto}^{p}$ is not closed, then it has an accumulation point in $S_{\perp}^{p}$ and $S_{\perp}^{p} \neq \varnothing$.

Definition 1.3 A nonisolated type $p \in S(\emptyset)$ is symmetric if and only if $\mathrm{SI}_{p}$ is a symmetric binary relation on $p(M)$. Otherwise, $p$ is asymmetric.

Since semi-isolation is transitive, it follows that $p$ is asymmetric if and only if $\left(p(M), \mathrm{SI}_{p}\right)$ is a proper quasiorder (with infinite strictly increasing chains). Asymmetric types may exist even in an $\omega$-stable theory, so their existence, in general, does not imply the strict order property (examples of that kind can be found in Sudoplatov [7], [8] and Tanović [10]).

Remark 1.4 It is well known that the symmetry of semi-isolation implies the symmetry of isolation. We will sketch the proof of this fact.

(1) If $\operatorname{tp}(a / b)$ is isolated and $b \in \operatorname{Sem}(a)$, then $\operatorname{tp}(b / a)$ is isolated too. To prove this fact, choose $\varphi(x, b) \in \operatorname{tp}(a / b)$ witnessing the isolation and choose $\psi(a, y) \in \operatorname{tp}(b / a)$ witnessing the semi-isolation. Then $\psi(a, y) \wedge \varphi(a, y) \vdash \operatorname{tp}(b / a)$. If $b^{\prime}$ satisfies this formula, then $\models \psi\left(a, b^{\prime}\right)$ implies $\operatorname{tp}\left(b^{\prime}\right)=\operatorname{tp}(b)$. Combining with $\models \varphi\left(a, b^{\prime}\right)($ and $\varphi(x, b) \vdash \operatorname{tp}(a / b))$, we derive $\operatorname{tp}\left(a b^{\prime}\right)=\operatorname{tp}(a b) ; \operatorname{tp}(b / a)$ is isolated.

(2) Suppose that $\operatorname{tp}(a / b)$ is isolated and that $\operatorname{tp}(b / a)$ is nonisolated. Then $b \rightarrow a$ and, by part (1), $b \notin \operatorname{Sem}(a)$. This shows that the asymmetry of isolation on a pair of elements implies the asymmetry of semi-isolation on the same pair. In particular, if $p \in S(\emptyset)$ and there are $a, b \models p$ such that $\operatorname{tp}(a / b)$ is isolated and $\operatorname{tp}(b / a)$ is nonisolated, then $p$ is asymmetric.

(3) Suppose that $\operatorname{tp}(a / b)$ is isolated. By part (1) we have

$$
\operatorname{tp}(b / a) \text { is nonisolated iff } b \notin \operatorname{Sem}(a) \text { iff } b \mapsto a .
$$

We will use a version of Remark 1.4 localized to $p$ : if semi-isolation is symmetric on $p(M)$, then isolation is symmetric on $p(M)$ too. The following example shows that the converse is not true: symmetry of isolation on $p(M)$ does not necessarily imply the symmetry of semi-isolation on $p(M)$.

Example 1.5 Let $T=\operatorname{Th}(\omega,<)$. Here there is a unique nonalgebraic 1-type $p(x)$ over $\emptyset$ (the type of an infinite element). Any infinite element has an immediate successor and a predecessor, so $x \pm n$ are well-defined functions and

$$
\mathrm{SI}_{p}=\bigcup_{n \in \omega}\left\{(x, y) \in p(M)^{2} \mid x-n<y\right\}
$$

(note that $x+n<y$ implies $x<y$ ). We have that $p$ is asymmetric. Take $a, b$ realizing $p$ such that $a+n<b$ holds for all integers $n$; then $a \mapsto b$. On the other hand, isolation on $p(M)$ is symmetric because it is witnessed by a formula of the form $x=y \pm n$ for some $n$.

Note that $\mathrm{SI}_{p}$ is not relatively definable within $p(M)^{2}$ because the union is strictly increasing. On the other hand, $\overline{\mathrm{SI}}_{p}=p(M)^{2}$ is obviously relatively definable within $p(M)^{2}$. Therefore there are asymmetric types for which $\overline{\mathrm{SI}}_{p}$ is relatively definable, while $\mathrm{SI}_{p}$ is not relatively definable within the locus. 
Recall that a nonisolated type $p \in S(\emptyset)$ is called powerful if the model prime over a realization of $p$ is weakly saturated (realizes all finitary types over $\emptyset$ ). Benda in [2] proved that powerful types exist in any Ehrenfeucht theory. Consider all the (isomorphism types of) countable models atomic over a finite subset, and order them by elementary embeddability. Then there is a maximal element (since there are finitely many isomorphism types); the maximal models are precisely those that are weakly saturated.

Remark 1.6 We note some well-known facts about powerful types. We sketch their proofs for the reader's convenience.

(1) Any powerful type is asymmetric. Let $p(x)$ be powerful, and let $a \models p$. Since $p$ is nonisolated, we can find $a^{\prime}$ realizing a nonisolated extension of $p$ in $S(a)$. Further, because $\operatorname{tp}\left(a a^{\prime}\right)$ is realized in any maximal model, there is $b \models p$ such that $\operatorname{tp}\left(a a^{\prime} / b\right)$ is isolated. Note that $\operatorname{tp}\left(a^{\prime} / a b\right)$ is isolated. If $\operatorname{tp}(b / a)$ were isolated, then by transitivity of isolation, $\operatorname{tp}\left(a^{\prime} b / a\right)$ would be isolated too. The latter implies isolation of $\operatorname{tp}\left(a^{\prime} / a\right)$, which is a contradiction. Therefore $\operatorname{tp}(b / a)$ is nonisolated while $\operatorname{tp}(a / b)$ is isolated, so isolation is asymmetric on $p(M)$. By Remark 1.4(2), we conclude that $p$ is asymmetric.

(2) Let $p$ be powerful. Then the proof of part (1) shows that for any $a \models p$ there exists $b \models p$ such that $b \mapsto a$.

(3) Semi-isolation is a downwards-directed quasiorder on the locus of a powerful type. If $a, b$ realize $p$, then by maximality there is $d$ realizing $p$ such that $\operatorname{tp}(a b / d)$ is isolated. In particular, $\operatorname{tp}(a / d)$ and $\operatorname{tp}(b / d)$ are isolated, by $\varphi(d, x)$ and $\psi(d, y)$, say, and we have $d \stackrel{\varphi}{\rightarrow} a$ and $d \stackrel{\psi}{\rightarrow} b$. We have that $d$ is a lower bound for $a$ and $b$.

By a $p$-principal formula we mean an $L$-formula $\varphi(x, y)$ such that for some (any) $a$ realizing $p$,

$\varphi(a, x)$ isolates an extension of $p$ in $S_{1}(a)$ and $a \stackrel{\varphi}{\mapsto} b$ holds for all $b \in \varphi(a, M)$.

By Remark 1.4(3), the condition $a \stackrel{\varphi}{\mapsto} b$ can be replaced by “ $\operatorname{tp}(a / b)$ is nonisolated."

Remark 1.7 Suppose that $p$ is powerful. We strengthen the conclusion of Remark 1.6(3): for all $a, b \in p(M)$ there is $d \in p(M)$ and $p$-principal formulas $\varphi$ and $\psi$ such that both $d \stackrel{\varphi}{\mapsto} a$ and $d \stackrel{\psi}{\mapsto} b$ hold. To prove it, first choose $c_{a}, c_{b} \models p$ satisfying $c_{a} \mapsto a$ and $c_{b} \mapsto b$ (here we use Remark 1.6(2)). Then choose $d \models p$ such that $\operatorname{tp}\left(c_{a} c_{b} a b / d\right)$ is isolated. Then $\operatorname{tp}\left(c_{a} / d\right)$ is isolated, by $\varphi(d, x)$, say. Further, $d \rightarrow c_{a} \mapsto a$ implies $d \mapsto a$ and $d \stackrel{\varphi}{\mapsto} a$. Similarly, $d \stackrel{\psi}{\mapsto} b$ for a suitably chosen $\psi$.

Recall that a theory $T$ is binary if every formula is equivalent modulo $T$ to a Boolean combination of formulas with at most two free variables. Binary theories are a special case of $\Delta$-based theories (see Saffe, Palyutin, and Starchenko [6]). There $\Delta$ is a fixed set of formulas (without parameters), and every formula without parameters is equivalent to a Boolean combination of formulas from $\Delta$. As noted in [6], this means precisely that any complete type $p \in S(\emptyset)$ is $\Delta$-based, that is, that $p$ is forced by the set of formulas $\varphi^{\delta} \in p$, where $\varphi \in \Delta$ and $\delta \in\{0,1\}$. In particular, a theory is binary if and only if any complete type is forced by the union of its 2-subtypes. 


\section{Definability of Semi-Isolation}

In this section we study definability properties of semi-isolation on the locus of an asymmetric type $p \in S(\emptyset)$. We know that $\mathrm{SI}_{p}$ is $\bigvee$-definable within $p(M)^{2}$. We will prove that certain additional assumptions on the topological complexity of $S_{p, p}$ imply the strict order property. The ordering relation found will always be a subset of $\mathrm{SI}_{p}$, as formalized in the next definition.

Definition 2.1 Suppose that $p \in S(\emptyset)$ and that $(p(M), \leq)$ is a quasiorder with infinite strictly increasing chains. We will say that $\leq$ is a $p$-order if

(1) $\leq$ is a relatively definable subset of $p(M)^{2}$, and

(2) $a \leq b$ implies $(a, b) \in \mathrm{SI}_{p}$.

The next proposition shows that a $p$-order is the restriction of a definable quasiorder to $p(M)$; the domain of such a quasiorder can be chosen to be definable and unbounded (contains no maximal elements).

Proposition 2.2 Suppose that $p \in S(\emptyset),(p(M), \leq)$ is a $p$-order, and that $\varphi(x, y)$ relatively defines $\leq$ within $p(M)^{2}$. Then there exists $\theta(x) \in p$ such that the formula $\theta(x) \wedge \theta(y) \wedge \varphi(x, y)$ witnesses $p$-semi-isolation and defines an unbounded quasiorder on $\theta(M)$.

Proof Denote by $\tau(x, y, z)$ the formula $\varphi(x, x) \wedge(\varphi(x, y) \wedge \varphi(y, z) \Rightarrow \varphi(x, z))$.

The first condition from the definition of a $p$-order implies

$$
p(x) \cup p(y) \cup p(z) \vdash \tau(x, y, z) .
$$

The second can be expressed by

$$
p(x) \cup p(y) \cup\{\varphi(x, y)\} \vdash \bigvee_{i \in I} \varphi_{i}(x, y),
$$

where the disjunction is taken over all formulas witnessing $p$-semi-isolation. By compactness there exists a finite $I_{0} \subset I$ such that (2.2) holds with $I_{0}$ in place of $I$. Then

$$
p(x) \cup p(y) \cup\{\varphi(x, y)\} \vdash \varphi(x, y),
$$

where $\varphi(x, y)$ is the formula $\bigvee_{i \in I_{0}} \varphi_{i}(x, y)$. Note that $\varphi(x, y)$ witnesses $p$-semiisolation. Now we apply compactness simultaneously to (2.1) and (2.3): there exists a formula $\theta_{0}(x)$ such that

$$
\theta_{0}(x) \wedge \theta_{0}(y) \wedge \theta_{0}(z) \vdash \tau(x, y, z) \quad \text { and } \quad \theta_{0}(x) \wedge \theta_{0}(y) \wedge \varphi(x, y) \vdash \varphi(x, y) \text {. }
$$

The first relation here implies that $\varphi(x, y)$ defines a quasi-order $\leq_{\varphi}$ on $\theta_{0}(M)$; its restriction to $p(M)$ is $\leq$. The second implies that $\theta_{0}(x) \wedge \theta_{0}(y) \wedge \varphi(x, y)$ witnesses $p$-semi-isolation. Now we show that there is no $\leq_{\varphi}$-maximal element in $\theta_{0}(M)$ above $a \in p(M)$. We have that $a \leq_{\varphi} b$ implies $b \in p(M)$ and, because $\leq$ is a $p$-order, there exists a strictly $\leq$-increasing chain above $b$. Thus $b$ is not $\leq$-maximal. But $\leq$ is a restriction of $\leq_{\varphi}$, so $b$ is not $\leq_{\varphi}$-maximal.

Let $\theta(x)$ be the conjunction of $\theta_{0}(x)$ and the formula saying that there is no $\leq_{\varphi}$-maximal element above $x$. Clearly, $\theta(x) \wedge \theta(y) \wedge \varphi(x, y)$ witnesses $p$-semiisolation and defines the restriction of $\leq_{\varphi}$ on $\theta(M)$. To finish the proof it remains to show that the restricted quasiorder is unbounded; this holds because $\theta(M)$ is $\leq_{\varphi}$-closed upwards in $\theta_{0}(M)$ and $\theta_{0}(M)$ is unbounded.

As an immediate corollary we obtain the following. 
Corollary 2.3 If $p(x) \in S(\emptyset)$ is asymmetric and $\mathrm{SI}_{p}$ is a relatively definable subset of $p(M)^{2}$, then there is $\theta(x) \in p$ and a definable, unbounded quasiorder on $\theta(M)$ whose restriction to $p(M)$ is $\mathrm{SI}_{p}$. In particular, $T$ has the strict order property.

This fact is well known and can be found in different forms in [1], [3], [5], and Tanović [9]. An example of an asymmetric type with relatively definable semiisolation is the unique nonisolated 1-type in Ehrenfeucht's example. A similar situation appears in any almost $\boldsymbol{\aleph}_{0}$-categorical theory: recall that $T$ is almost $\aleph_{0}$-categorical (see [3]) if $p_{1}\left(x_{1}\right) \cup p_{2}\left(x_{2}\right) \cup \cdots \cup p_{n}\left(x_{n}\right)$ has only finitely many completions $r\left(x_{1}, \ldots, x_{n}\right) \in S(\emptyset)$ for all $n$ and all complete types $p_{i}\left(x_{i}\right) \in S(\emptyset)$. For any $p$ in such a theory, $\mathrm{SI}_{p}$ is relatively definable within $p(M)^{2}: S_{p, p}$ is finite, so all its relevant parts are clopen, and by Remark $1.2(7), \mathrm{SI}_{p}$ is relatively definable; alternatively, there are only finitely many inequivalent formulas witnessing $p$-semi-isolation, so their disjunction relatively defines $\mathrm{SI}_{p}$ within $p(M)^{2}$.

Corollary 2.4 If $p(x) \in S(\emptyset)$ is asymmetric and $S_{p, p}$ is finite, then there is $\theta(x) \in p$ and a definable, unbounded quasiorder on $\theta(M)$ whose restriction to $p(M)$ is $\mathrm{SI}_{p}$. In particular, $T$ has the strict order property.

Example 2.5 Let $T=\left(\mathbb{Q},<, c_{n}, d_{n}\right)$, where $\left(c_{n}\right)$ is an increasing and $\left(d_{n}\right)$ is a decreasing sequence such that both converge to $\sqrt{2}$. We have that $T$ is an Ehrenfeucht theory having six countable models. Let $p$ be the 1-type representing $\sqrt{2}$. Then the locus of $p$ is convex and linearly ordered by $<$. However, $p$ is symmetric and $\mathrm{SI}_{p}$ is the identity relation. Thus there is no $p$-order there!

Therefore, the locus of a symmetric type may be properly ordered and the asymmetry of semi-isolation is not an exclusive reason for the presence of the strict order property. However, we believe that in this example the reason for the absence of $p$-orders lies in nonpowerfulness of $p$.

Question 2 Suppose that $p$ is a powerful type in an Ehrenfeucht theory. Does the existence of a nontrivial, relatively definable, partial order on $p(M)$ always imply the existence of a $p$-order?

It is easy to realize that relative definability of $\mathrm{SI}_{p}$ implies relative definability of $\overline{\mathrm{SI}}_{p}$ within $p(M)^{2}$. The converse is, in general, not true as Example 1.5 shows. There the asymmetric type $p \in S_{1}(\emptyset)$ is such that $\overline{\mathrm{SI}}_{p}$ is relatively definable within $p(M)^{2}$, while $\mathrm{SI}_{p}$ is not.

We will prove in Corollary 2.8 below that relative definability of $\overline{\mathrm{SI}}_{p}$ for asymmetric $p$ implies the existence of a $p$-order. Actually, the order found in the proof will have an additional property which will witness that semi-isolation is partially definable on $p(M)$. This notion was introduced in [10], and here we give an equivalent definition which relies on the notion of a $p$-order.

Definition 2.6 We say that semi-isolation is partially definable on $p$ if there is a definable quasi-order $\leq$ such that for all $a \in p(M)$,

(i) the restriction of $\leq$ to $p(M)$ is a $p$-order, and

(ii) $a \stackrel{\leq}{\mapsto} b \rightarrow b^{\prime}$ and $b^{\prime} \in p(M)$ imply $a \stackrel{\leq}{\mapsto} b^{\prime}$.

Clearly, partial definability of semi-isolation implies that $T$ has the strict order property. 
Question 3 Does the existence of a $p$-order imply partial definability of semiisolation on $p$ ?

Theorem 2.7 Suppose that $p \in S(\emptyset)$ is asymmetric and that $S_{\mapsto}^{p}$ is closed in $S_{p, p}$. Then semi-isolation is partially definable on $p(M)$. In particular, $T$ has the strict order property.

Proof Suppose that $S_{\mapsto}^{p}$ is closed in $S_{p, p}$. Then it is compact. For each $q(x, y) \in S_{\mapsto}^{p}$, choose a formula $\varphi_{q}(x, y) \in q(x, y)$ witnessing $p$-semi-isolation. Then $S_{\mapsto}^{p} \subseteq \bigcup\left\{\left[\varphi_{q}\right] \mid q \in S_{\mapsto}^{p}\right\}$. Since $S_{\mapsto}^{p}$ is compact, there is a finite subcover. Let $\varphi(x, y)$ be the disjunction of all the $\varphi_{q}$ 's from the subcover. Then $\varphi$ witnesses $p$-semi-isolation and $S_{\mapsto}^{p} \subseteq[\varphi] \subseteq S_{\rightarrow}^{p}$ holds. Let $x \leq y$ be

$$
x=y \vee(\varphi(x, y) \wedge(\forall t)(\varphi(y, t) \Rightarrow \varphi(x, t))) .
$$

Clearly, $\leq$ defines a quasiorder on $M$; it also witnesses $p$-semi-isolation.

Claim 1 If $a \mapsto b$ realize $p$, then $\varphi(b, M) \subsetneq \varphi(a, M)$ and $a<b$.

Proof Suppose that $d \in \varphi(b, M)$. Then $a \mapsto b \rightarrow d$ implies $a \mapsto d$ and $\operatorname{tp}(a d) \in S_{\mapsto}^{p} \subseteq[\varphi]$. Thus $d \in \varphi(a, M)$ and $\varphi(b, M) \subsetneq \varphi(a, M)$ holds. Similarly, $a \mapsto b$ implies $\operatorname{tp}(a b) \in S_{\mapsto}^{p} \subseteq[\varphi]$, so $\models \varphi(a, b)$. Finally, $\models \varphi(a, b)$ and $\varphi(b, M) \subsetneq \varphi(a, M)$ imply $a<b$.

Since $p$ is asymmetric, no element of $p$ is maximal in the semi-isolation quasiorder. Then, by the claim, no realization of $p$ is $\leq$-maximal. We conclude that $\leq$ defines a $p$-order on $p(M)$, proving condition (i) from the definition of partial semi-isolation. To prove (ii), suppose that $a \stackrel{\leq}{\mapsto} b \rightarrow c$ holds. Then $a \mapsto c$ and the claim implies $a<c$. Therefore $a \stackrel{\leq}{\mapsto} c$ holds, proving (ii). The symbol $\leq$ partially defines semi-isolation on $p$.

Corollary 2.8 Suppose that $p(x) \in S(\emptyset)$ is asymmetric and that $\overline{\mathrm{SI}}_{p}$ is a relatively definable subset of $p(M)^{2}$. Then semi-isolation is partially definable on $p(M)$. In particular, $T$ has the strict order property.

Proof Suppose that $\overline{\mathrm{SI}}_{p}$ is relatively definable within $p(M)^{2}$. We will show that $S_{\mapsto}^{p}$ is closed in $S_{p, p}$. By Remark 1.2(8) $S_{\rightarrow}^{p} \cup S_{\leftarrow}^{p}$ is closed; clearly it contains $S_{\mapsto}^{p}$, so $\operatorname{cl}\left(S_{\mapsto}^{p}\right) \subseteq S_{\rightarrow}^{p} \cup S_{\leftarrow}^{p}$. On the other hand, by Remark 1.2(9) we have $\operatorname{cl}\left(S_{\mapsto}^{p}\right) \subseteq S_{\mapsto}^{p} \cup S_{\perp}^{p}$. Therefore

$$
\operatorname{cl}\left(S_{\mapsto}^{p}\right) \subseteq\left(S_{\rightarrow}^{p} \cup S_{\leftarrow}^{p}\right) \cap\left(S_{\mapsto}^{p} \cup S_{\perp}^{p}\right)=S_{\mapsto}^{p} .
$$

Therefore $S_{\mapsto}^{p}$ is closed in $S_{p, p}$, and the conclusion follows by Theorem 2.7.

Corollary 2.9 ( $T$ is NSOP) If $p \in S(\emptyset)$ is asymmetric, then $S_{\mapsto}^{p}$ (is infinite and) has an accumulation point in $S_{\perp}^{p}$. In particular, $S_{\perp}^{p} \neq \emptyset$ and $p(x) \cup p(y) \cup x \perp{ }^{p} y$ is consistent.

Proof By Remark 1.2(9) we have $\operatorname{cl}\left(S_{\mapsto}^{p}\right) \subseteq S_{\mapsto}^{p} \cup S_{\perp}^{p}$. The NSOP assumption combined with Theorem 2.7 implies that $S_{\mapsto}^{p}$ is not closed in $S_{p, p}$, so there exists $q \in \operatorname{cl}\left(S_{\mapsto}^{p}\right) \backslash S_{\mapsto}^{p}$. Then $q$ is an accumulation point of $S_{\mapsto}^{p}$ and $q \in S_{\perp}^{p}$. In particular, $S_{\perp}^{p} \neq \emptyset$, so $p(x) \cup p(y) \cup x \perp^{p} y$ is consistent. 
Theories with few links were introduced by Benda in [2]: $T$ has few links if whenever $p(\bar{x})$ and $q(\bar{y})$ are complete types, then there are only finitely many complete types $r(\bar{x}, \bar{y}) \supset p(\bar{x}) \cup q(\bar{y})$ such that $r(\bar{c}, \bar{y})$ is nonisolated in $S(\bar{c})$ for all $\bar{c}$ realizing $p(\bar{x})$. Pillay in [5, Theorem 5] proved that any Ehrenfeucht theory with few links has the strict order property. He noted that his proof uses only the assumption when $p=q$ is a powerful type. Indeed, it is not hard to realize that the few-links assumption implies that $S_{\mapsto}^{p}$ is finite for any $p \in S(\emptyset)$ : If $\bar{a}, \bar{b} \models p$ and $\bar{a} \mapsto \bar{b}$, then $\operatorname{tp}(\bar{a} / \bar{b})$ is nonisolated; there are only finitely many possibilities for $\operatorname{tp}(\bar{a} / \bar{b})$, so $S_{\mapsto}^{p}$ is finite. In particular, $S_{\mapsto}^{p}$ is closed in $S_{p, p}$, and we have the following.

Corollary 2.10 Any theory with few links and an asymmetric type has the strict order property.

In the same article, Pillay [5, Section 6] commented on the few-links assumption: "This condition is admittedly rather artificial, but it enables some proofs to go through." An easy consequence of the few-links assumption is that $\mathrm{CB}\left(S_{p, p}\right) \leq 1$ holds for all $p \in S(\emptyset)$ (simply because $S_{p, p}$ cannot have infinitely many accumulation points). So $\operatorname{CB}\left(S_{p, p}\right)=1$ seems to be a more natural condition. There are such Ehrenfeucht theories, the first example having been found by Woodrow in [13].

Question 4 Is there a powerful type $p$ in an NSOP theory satisfying $\mathrm{CB}\left(S_{p, p}\right)=1$ ?

In this article, we do not give much evidence towards answering this question.

Corollary 2.11 ( $T$ is small, NSOP) Suppose that $p \in S(\emptyset)$ is asymmetric (not necessarily powerful) and that $\mathrm{CB}\left(S_{p, p}\right)=1$ holds. Then

(1) $\left|S_{\mapsto}^{p}\right| \geq \aleph_{0}$ and $\left|S_{\perp}^{p}\right| \geq 1$, and

(2) there are infinitely many pairwise inequivalent p-principal formulas.

Proof Condition (1) follows from Corollary 2.9. To prove (2), note that $\mathrm{CB}\left(S_{p, p}\right)=1$ implies that there are infinitely many members of $S_{\mapsto}^{p}$ isolated in $S_{p, p}$. If $\operatorname{tp}(a b) \in S_{\mapsto}^{p}$ is such a type, then $\operatorname{tp}(b / a)$ is isolated and contains a $p$-principal formula.

\section{Incomparability}

In this section, we start dealing with the $\mathrm{SI}_{p}$-incomparability of realizations of an asymmetric type. By Corollary 2.9, it is an interesting relation especially in NSOP theories. The next theorem deals with the case when $\overline{\mathrm{SI}}_{p}$ has relatively definable intersection with the product of two relatively definable subsets of $p(M)$. We will prove that there is a pair of incomparable elements $(a, b) \in D_{1} \times D_{2}$. The intended combinatorial description of this situation is formalized in Proposition 4.3: if we have two large, unbounded, relatively definable subsets of $p(M)$, then some pair of their elements is incomparable.

Theorem 3.1 Suppose that $p \in S_{1}(\emptyset)$ is nonisolated and that $D_{1}, D_{2} \subset M$ are $\bar{e}$-definable subsets of $M$ such that the following conditions are satisfied.

(1) $\overline{\mathrm{SI}}_{p} \cap\left(D_{1} \times D_{2}\right) \neq \emptyset$ is relatively $\bar{e}$-definable within $D_{1} \times D_{2}$.

(2) For all $a \in D_{1} \cap p(M)$ there is $b \in D_{2} \cap p(M)$ such that $a \mapsto b$.

(3) For all $b \in D_{2} \cap p(M)$ there is $a \in D_{1} \cap p(M)$ such that $b \rightarrow a$. 
Then there is an $\bar{e}$-definable quasiorder on $M$ such that no element of $D_{1} \cap p(M)$ is below a maximal one of $D_{1}$. In particular, $T$ has the strict order property.

Proof Suppose that $D_{i}$ is defined by $D_{i}(x, \bar{e})$ and that relative definability is witnessed by $\theta(x, y, \bar{e})$. So we have

$$
p(x) \cup p(y) \cup\left\{D_{1}(x, \bar{e}), D_{2}(y, \bar{e}), \theta(x, y, \bar{e})\right\} \vdash y \in \operatorname{Sem}_{p}(x) \vee x \in \operatorname{Sem}_{p}(y) .
$$

The right-hand side is a long disjunction, so by compactness there is an $L$-formula $\varphi(x, y)$ witnessing $y \in \operatorname{Sem}_{p}(x)$ and there is an $L$-formula $\psi^{*}(x, y)$ witnessing $x \in \operatorname{Sem}_{p}(y)$ such that

$$
p(x) \cup p(y) \cup\left\{D_{1}(x, \bar{e}), D_{2}(y, \bar{e}), \theta(x, y, \bar{e})\right\} \vdash \varphi(x, y) \vee \psi^{*}(y, x) .
$$

Let $\psi(x, y):=\psi^{*}(y, x)$. Then for any pair $(a, b) \in D_{1} \times D_{2}$ of realizations of $p$, we have

$$
\text { either } \models \neg \theta(a, b, \bar{e}) \text { or: at least one of } a \stackrel{\varphi}{\rightarrow} b \text { and } b \stackrel{\psi}{\rightarrow} a \text { holds. }
$$

(The first disjunction here is exclusive because $\theta(x, y, \bar{e})$ relatively defines $\overline{\mathrm{SI}}_{p} \cap$ $D_{1} \times D_{2}$.) Further, we express assumption (3) by

$$
p(x) \cup\left\{D_{2}(x, \bar{e})\right\} \vdash \bigvee_{\psi^{\prime}(x, y)} \exists y\left(D_{1}(y, \bar{e}) \wedge \psi^{\prime}(x, y)\right),
$$

where the disjunction is taken over all $\psi^{\prime}(x, y)$ witnessing $p$-semi-isolation. By compactness, for some $\psi^{\prime}(x, y)$ we have

for all $b \in D_{2} \cap p(M)$ there is $c \in D_{1} \cap p(M)$ such that $b \stackrel{\psi^{\prime}}{\rightarrow} c$ holds.

After replacing both $\psi$ and $\psi^{\prime}$ by their disjunction, we may assume that $\psi=\psi^{\prime}$. Let $\varphi(x, y, \bar{e})$ be $\exists z\left(D_{2}(z, \bar{e}) \wedge \varphi(x, z) \wedge \psi(z, y)\right)$. Then $\varphi(a, y, \bar{e}) \vdash p(y)$ for any $a$ realizing $p$.

Claim 1 For any $a \in D_{1} \cap p(M)$, there exists $c \in D_{1}$ satisfying $a \mapsto c$ and $\models \varphi(a, c, \bar{e})$.

Proof Let $a \in D_{1} \cap p(M)$. By (3.2) there is $b \in D_{2} \cap p(M)$, and by (3.3) there is $c \in D_{1} \cap p(M)$ such that $a \mapsto b \stackrel{\psi}{\rightarrow} c$ holds. Then $(a, b) \in \overline{\operatorname{SI}}_{p}$ implies $\models \theta(a, b, \bar{e})$, and $a \notin \operatorname{Sem}_{p}(b)$ implies that $b \stackrel{\psi}{\rightarrow} a$ does not hold. By (3.1) we derive $a \stackrel{\varphi}{\mapsto} b$. Thus $a \stackrel{\varphi}{\mapsto} b \stackrel{\psi}{\rightarrow} c$, and so $\models \varphi(a, c, \bar{e})$.

Define $a^{\prime} \leq b^{\prime}$ iff $\varphi\left(b^{\prime}, M, \bar{e}\right) \cap D_{1} \subseteq \varphi\left(a^{\prime}, M, \bar{e}\right) \cap D_{1}$. Clearly, $\leq$ is a definable quasiorder on $M$. We will show that no element of $D_{1} \cap p(M)$ is below a maximal one of $D_{1}$.

Claim 2 If $a, c \in D_{1} \cap p(M)$ and $a \mapsto c$, then $a \leq c$.

Proof Suppose that $d \in \varphi(c, M, \bar{e}) \cap D_{1}$, and let $b \in D_{2}$ be such that $c \stackrel{\varphi}{\rightarrow} b \stackrel{\psi}{\rightarrow} d$. Then $a \mapsto c \rightarrow b$ implies $a \mapsto b$, so $b \stackrel{\psi}{\rightarrow} a$ does not hold; also, $(a, b) \in \overline{\mathrm{SI}}_{p}$ implies $\models \theta(a, b, \bar{e})$. By (3.1) we conclude that $a \stackrel{\varphi}{\mapsto} b$ holds, and then $a \stackrel{\varphi}{\mapsto} b \stackrel{\psi}{\rightarrow} d$ implies $\varphi(a, d, \bar{e})$. Thus $d \in \varphi(a, M, \bar{e})$. This shows that $\varphi(c, M, \bar{e}) \cap D_{1} \subseteq \varphi(a, M, \bar{e}) \cap D_{1}$; that is, $a \leq c$. 
Now, let $a_{1} \in D_{1} \cap p(M)$. By Claim 1 there is $c_{1} \in D_{1}$ such that $a_{1} \mapsto c_{1}$ and $\models \varphi\left(a_{1}, c_{1}, \bar{e}\right)$. By Claim 2 we have $a_{1} \leq c_{1}$. Repeating the same procedure with $c_{1}$, we find $a_{2} \in D_{1}$ satisfying $c_{1} \mapsto a_{2}, \models \varphi\left(c_{1}, a_{2}, \bar{e}\right)$, and $c_{1} \leq a_{2}$. In particular, $a_{1} \leq a_{2}$; that is, $\varphi\left(a_{2}, M, \bar{e}\right) \cap D_{1} \subseteq \varphi\left(a_{1}, M, \bar{e}\right) \cap D_{1}$. Then $c_{1} \notin \varphi\left(a_{2}, M, \bar{e}\right)$; otherwise $\models \varphi\left(a_{2}, c_{1}, \bar{e}\right)$ would witness $a_{2} \rightarrow c_{1}$, which is in contradiction with $c_{1} \mapsto a_{2}$. Thus $c_{1} \in \varphi\left(a_{1}, M, \bar{e}\right) \backslash \varphi\left(a_{2}, M, \bar{e}\right)$ and $a_{1}<a_{2}$. Continuing in this way we get an infinite strictly increasing chain of elements of $D_{1} \cap p(M)$.

\section{Semi-Isolation on Minimal Powerful Types}

Throughout this section we will assume that $T$ (is small and) has a powerful type. We will say that $p \in S(\emptyset)$ is a minimal powerful type if it is powerful and there is a formula $\theta(x) \in p$ such that $p$ is the unique powerful type containing $\theta$. Minimal powerful types exist in any Ehrenfeucht theory-take a powerful type of minimal CB-rank. To simplify notation, unless otherwise stated we will assume that $p \in S_{1}(\emptyset)$ is powerful.

We will be interested in sets definable over a single parameter; we do not a priori assume that the parameter realizes even a nonisolated type. We will say that $D=\varphi(d, M)$ is a $p$-set if $D \cap p(M) \neq \varnothing$ and there exists $b \in D \cap p(M)$ such that at least one of the following two conditions holds:

1. $b$ does not semi-isolate $d$;

2. $\operatorname{tp}(d)$ is not powerful.

The intended intuitive description of a $p$-set is that $D \cap p(M)$ is large and unbounded; this is formalized in Lemma 4.2 below.

Remark 4.1 Suppose that $p$ is a powerful type.

(1) If $\operatorname{tp}(d)$ is not powerful, then the second condition from the definition of a $p$-set is satisfied, so $D=\varphi(d, M)$ is a $p$-set if and only if it contains a realization of $p$.

(2) Suppose that $p$ is a minimal powerful type and that $\theta(x) \in p$ witnesses the minimality. Let $d \in \theta(M) \backslash p(M)$. Then, by part $(1), D=\varphi(d, M)$ is a $p$-set whenever it contains a realization of $p$.

(3) Suppose that $d \models p$ and that $\varphi(x, y)$ witnesses the asymmetry of $p$-semiisolation; there are $a, b \in p(M)$ such that $a \stackrel{\varphi}{\mapsto} b$. Then $b$ witnesses that the first condition from the definition holds for $D=\varphi(a, M)$, so $\varphi(a, M)$ is a $p$-set. In particular, $\psi(a, M)$ is a $p$-set for any $p$-principal formula $\psi(x, y)$ and $a \models p$.

(4) Suppose that $p$ is a minimal powerful type and that the minimality is witnessed by $\theta(x) \in p(x)$. If $\varphi(x, y)$ is a $p$-principal formula, then for all $d \in \theta(M), D=\varphi(d, M)$ is a $p$-set if and only if it contains a realization of $p$. For $d \in p(M)$ this follows from part (3), and for $d \notin p(M)$ from part (1).

Lemma 4.2 Suppose that $\theta(x) \in p(x)$ witnesses that $p \in S_{1}(\emptyset)$ is a minimal powerful type, $d \in \theta(M)$, and that $D=\varphi(d, M)$ is a p-set. Then $D \cap p(M)$ does not have an $\mathrm{SI}_{p}$-upper bound. 
Proof Suppose on the contrary that $a \in p(M)$ is an upper bound for $D \cap p(M)$. Then $c \rightarrow a$ holds for all $c \in D \cap p(M)$ :

$$
p(x) \cup\{\varphi(d, x)\} \vdash \bigvee_{\psi} \psi(x, a) .
$$

By compactness there are $\theta_{0}(x) \in p(x)$ (wlog implying $\left.\theta(x)\right)$ and $\psi(x, y)$ witnessing $p$-semi-isolation such that $\models\left(\theta_{0}(x) \wedge \varphi(d, x)\right) \Rightarrow \psi(x, a)$. Define

$$
\sigma(y, z):=\forall t\left(\left(\theta_{0}(t) \wedge \varphi(y, t)\right) \Rightarrow \psi(t, z)\right) .
$$

Then $\models \sigma(d, a)$ holds, and according to the definition we have two cases.

Case 1 There exists $b \in D \cap p(M)$ such that $b$ does not semi-isolate $d$.

In this case, we have

$$
\models \varphi(d, b) \wedge \theta(d) \wedge \exists z \sigma(d, z) .
$$

Since $b$ does not semi-isolate $d$, any formula from $\operatorname{tp}(d / b)$ is consistent with infinitely many types from $S_{1}(\emptyset)$, so there exists $d^{\prime} \in M$ which does not realize $p$ and satisfies (4.1) in place of $d$. Note that $\models \theta\left(d^{\prime}\right)$ and the minimality of $p$ together imply that $\operatorname{tp}\left(d^{\prime}\right)$ is not powerful. Let $a^{\prime}$ be such that

$$
\vDash \varphi\left(d^{\prime}, b\right) \wedge \theta\left(d^{\prime}\right) \wedge \sigma\left(d^{\prime}, a^{\prime}\right) .
$$

We claim that $\sigma\left(d^{\prime}, z\right) \vdash p(z)$ holds. Assume $\models \sigma\left(d^{\prime}, c\right)$. Then from $b \in \theta_{0}(M) \cap$ $\varphi\left(d^{\prime}, M\right)$ and the definition of $\sigma$, we get $\models \psi(b, c)$. Since $\psi$ witnesses $p$-semiisolation, the claim follows.

$T$ is small, so there is an isolated type in $S_{1}\left(d^{\prime}\right)$ containing $\sigma\left(d^{\prime}, t\right)$ (it is an extension of $p$ ). Thus $d^{\prime}$ isolates an extension of $p$, and because $p$ is powerful, $\operatorname{tp}\left(d^{\prime}\right)$ has to be powerful too. This is a contradiction.

Case $2 \operatorname{tp}(d)$ is not powerful.

Since $D$ is a $p$-set, there exists $b^{\prime} \in \varphi(d, M) \cap p(M)$. Assuming $\models \sigma\left(d, c^{\prime}\right)$ and arguing as in the first case, we derive $b^{\prime} \stackrel{\psi}{\rightarrow} c^{\prime}$, so $\sigma(d, z) \vdash p(z)$. Again, we can find an isolated extension of $p$ in $S_{1}(d)$ and conclude that $\operatorname{tp}(d)$ is powerful. This is a contradiction.

Next we show that $\mathrm{SI}_{p}$-incomparability appears quite often on the locus of a minimal powerful type in an NSOP theory.

Proposition 4.3 ( $T$ is NSOP) Suppose that $\theta(x) \in p(x)$ witnesses that $p$ is a minimal powerful type, $d_{i} \in \theta(M)$, and that each $D_{i}=\varphi_{i}\left(d_{i}, M\right)$ is a p-set for $i=1,2$. Then there are $a \in D_{1}, b \in D_{2}$ realizing $p$ such that $a \perp_{p} b$.

Proof Otherwise, for all $a \in D_{1}, b \in D_{2}$ realizing $p$ we have $(a, b) \in \overline{\mathrm{SI}}_{p}$, so

$$
\text { at least one of } a \rightarrow b \text { and } b \rightarrow a \text { holds. }
$$

In particular, $\overline{\mathrm{SI}}_{p} \cap\left(D_{1} \times D_{2}\right)$ is relatively $d_{1} d_{2}$-definable within $p(M)^{2}$, and the first assumption of Theorem 3.1 is satisfied. We will prove that the other two are satisfied too.

Suppose that the second condition fails, and witness the failure by $a \in D_{1} \cap p(M)$. Then, by (4.2), $b \rightarrow a$ would hold for all $b \in D_{2} \cap p(M)$, so $a$ would be an upper bound for $D_{2} \cap p(M)$; this is in contradiction with Lemma 4.2. Therefore the second and, similarly, the third condition are fulfilled. By Theorem 3.1, $T$ has the strict order property. This is a contradiction. 
Thus $\mathrm{SI}_{p}$ is in some sense a "wide" quasiorder. Because $p$ is powerful, it is also directed downwards. It is interesting to know whether it has to be directed upwards.

Question 5 Must $\mathrm{SI}_{p}$ be directed upwards on the locus of a minimal powerful type in an NSOP theory?

We have proved in Corollary 2.9 that $S_{\perp}^{p}$ has at least one element, and here, under much stronger assumptions, we will prove that $\left|S_{\perp}^{p}\right| \geq 2$.

Proposition 4.4 Suppose that $T$ is a binary NSOP theory with three countable models and that $p \in S_{1}(\emptyset)$ has CB-rank 1. Then $q(x, y)=p(x) \cup p(y) \cup x \perp_{p} y$ has at least two completions in $S_{2}(\varnothing)$.

Proof In a theory with three countable models there is a unique isomorphism type of a "middle model," that is, a countable model prime over a realization of a nonisolated type. The middle model is weakly saturated because every finitary type is realized in some finitely generated model. Thus any nonisolated type is powerful and, in particular, $p$ is powerful. Let $\theta(x) \in p$ be a formula of CB-rank 1 and CB-degree 1 . Then $p$ is the unique nonisolated type containing $\theta(x)$, and $p$ is a minimal powerful type.

$p$ is asymmetric, so by Corollary $2.9, q(x, y)$ is consistent. Now suppose that the conclusion of the proposition fails: $q(x, y)$ has a unique completion $q^{\prime}(x, y) \in S_{2}(\varnothing)$. Choose $a b \models q^{\prime}$; then $a \perp_{p} b$ holds. By Corollary 2.9, $q^{\prime}$ is an accumulation point of $S_{\mapsto}^{p}$, so each of $\operatorname{tp}(a b), \operatorname{tp}(a / b)$, and $\operatorname{tp}(b / a)$ is nonisolated. By the three model assumption, we know that the model prime over $a b$ is also prime over a realization $d$ of $p$ (because any two models prime over a realization of a nonisolated type are isomorphic). Note that both $\operatorname{tp}(a b / d)$ and $\operatorname{tp}(d / a b)$ are isolated. Hence there is a formula $\tau(x, y, z) \in \operatorname{tp}(d a b)$ such that $\tau(d, y, z)$ isolates $\operatorname{tp}_{y z}(a b / d)$ and $\tau(x, a, b)$ isolates $\operatorname{tp}_{x}(d / a b)$. Now we use the assumption that $T$ is binary: there are formulas $\varphi^{\prime}, \psi^{\prime}, \sigma$ such that

$$
\vDash\left(\varphi^{\prime}(x, y) \wedge \psi^{\prime}(x, z) \wedge \sigma(y, z)\right) \leftrightarrow \tau(x, y, z) .
$$

The assumed isolation properties of $\tau$ imply

$$
\begin{gathered}
\varphi^{\prime}(x, a) \wedge \psi^{\prime}(x, b) \wedge \sigma(a, b) \vdash p(x) ; \\
\varphi^{\prime}(d, y) \wedge \psi^{\prime}(d, z) \wedge \sigma(y, z) \vdash \operatorname{tp}(a b / d) .
\end{gathered}
$$

Let $\operatorname{tp}(a / d)$ be isolated by $\varphi(d, y) \in \operatorname{tp}(a / d)$, and let $\operatorname{tp}(b / d)$ be isolated by $\psi(d, z) \in \operatorname{tp}(b / d)$. Without loss of generality, assume that they are chosen so that $\vDash\left(\varphi(x, y) \Rightarrow \varphi^{\prime}(x, y)\right) \wedge\left(\psi(x, y) \Rightarrow \psi^{\prime}(x, y)\right)$. Then by (4.3) and (4.4):

$$
\begin{aligned}
& \varphi(x, a) \wedge \psi(x, b) \wedge \sigma(a, b) \vdash p(x) ; \\
& \varphi(d, y) \wedge \psi(d, z) \wedge \sigma(y, z) \vdash \operatorname{tp}(a b / d) .
\end{aligned}
$$

Now consider the formula $(\exists x)(\theta(x) \wedge \varphi(x, y) \wedge \psi(x, z) \wedge \sigma(y, z))$ which is in $\operatorname{tp}_{y z}(a b)=q^{\prime}(y, z)$. Since $S_{\perp}^{p}=\left\{q^{\prime}\right\}$, by Corollary 2.9, $q^{\prime}$ is an accumulation point of $S_{\mapsto}^{p}$, so there are $a^{\prime} b^{\prime}$ satisfying this formula such that $\operatorname{tp}\left(a^{\prime} b^{\prime}\right) \in S_{\mapsto}^{p}$; hence $\left(a^{\prime}, b^{\prime}\right) \in \mathrm{SI}_{p}$. Then for some $d^{\prime}$ we have

$$
\models \theta\left(d^{\prime}\right) \wedge \varphi\left(d^{\prime}, a^{\prime}\right) \wedge \psi\left(d^{\prime}, b^{\prime}\right) \wedge \sigma\left(a^{\prime}, b^{\prime}\right) .
$$

$d^{\prime}$ does not realize $p$; otherwise (4.6) would imply $a^{\prime} b^{\prime} \models q^{\prime}$, which is in contradiction with $\left(a^{\prime}, b^{\prime}\right) \in \mathrm{SI}_{p}$. Thus $d^{\prime} \in \theta(M) \backslash p(M)$, so by Remark 4.1(2), 
$D_{1}=\varphi\left(d^{\prime}, M\right)$ and $D_{2}=\psi\left(d^{\prime}, M\right)$ are $p$-sets. By Proposition 4.3, there are $a^{\prime \prime} \in D_{1}$ and $b^{\prime \prime} \in D_{2}$ realizing $p$ such that $a^{\prime \prime} \perp_{p} b^{\prime \prime}$ holds. The uniqueness of $q^{\prime}$ implies $a^{\prime \prime} b^{\prime \prime} \models q^{\prime}$ and $\models \sigma\left(a^{\prime \prime}, b^{\prime \prime}\right)$. Thus

$$
\models \varphi\left(d^{\prime}, a^{\prime \prime}\right) \wedge \psi\left(d^{\prime}, b^{\prime \prime}\right) \wedge \sigma\left(a^{\prime \prime}, b^{\prime \prime}\right) .
$$

By (4.5) and $\operatorname{tp}(a b)=\operatorname{tp}\left(a^{\prime \prime} b^{\prime \prime}\right)=q^{\prime}$, we get $d^{\prime} \models p$. This is a contradiction.

\section{PGPIP for Binary Theories}

Throughout this section we will assume that $T$ is a small, binary theory and that $p$ is a powerful 1-type. We have already noted in Remark 1.6 that $\mathrm{SI}_{p}$ is directed downwards. In Remark 1.7 we noted a stronger form: for any pair of elements $a, b \in p(M)$ there exists $d \in p(M)$ and $p$-principal formulas $\varphi, \psi$ such that both $d \stackrel{\varphi}{\rightarrow} a$ and $d \stackrel{\psi}{\rightarrow} b$ hold. In all the basic examples $\varphi$ and $\psi$ can be chosen from a finite (fixed in advance) set. This property is labeled in [8] as the global pairwise intersection property (GPIP) for $p$. Precisely, it means that there is a formula $\varphi(x, y)$ which is a disjunction of $p$-principal formulas and such that $\left(p(M), \varphi\left(M^{2}\right)\right)$ is an acyclic digraph satisfying

$$
\text { for all } a, b \in p(M) \text { there exists } d \models p \text { such that } \models \varphi(d, a) \wedge \varphi(d, b) .
$$

Here we introduce a somewhat stronger property.

Definition 5.1 $\quad p$ has PGPIP if there is a formula $\varphi(x, y)$ which is a disjunction of $p$-principal formulas and is such that $\left(p(M)^{2}, \varphi(M)\right)$ is an acyclic digraph, and for all $a, b \in p(M)$ there exists $d \models p$ satisfying

$$
\operatorname{tp}(a b / d) \text { is isolated and } \models \varphi(d, a) \wedge \varphi(d, b) .
$$

We leave it to the reader to check that nonisolated 1-types from Ehrenfeucht's and Peretyatkin's (see [4]) examples have PGPIP.

Theorem 5.2 ( $\boldsymbol{T}$ is binary, NSOP) Suppose that $\varphi(x, y)=\bigvee_{i=1}^{n} \varphi_{i}(x, y)$, where each $\varphi_{i}(x, y)$ is p-principal, witnesses PGPIP for $p$. Then $n \geq 2$ and $\mathrm{CB}\left(S_{p, p}(\emptyset)\right)<n^{2}$.

Proof Fix $d$ realizing $p$. For each pair $i, j \leq n$, define

$$
\begin{aligned}
& D_{(i, j)}=\left\{(a, b) \in p(M)^{2} \mid \operatorname{tp}(a b / d) \text { is isolated and } \models \varphi_{i}(d, a) \wedge \varphi_{j}(d, b)\right\}, \\
& C_{(i, j)}=\left\{\operatorname{tp}(a b / d) \mid(a, b) \in D_{(i, j)}\right\} \quad S_{(i, j)}=\left\{\operatorname{tp}(a b) \mid(a, b) \in D_{(i, j)}\right\} .
\end{aligned}
$$

Note that PGPIP implies that $\bigcup_{(i, j)} S_{(i, j)}=S_{p, p}(\emptyset)$ holds; in particular, if $n=1$, then $S_{(1,1)}=S_{p, p}(\emptyset)$.

Claim 1 For every $q(x, y) \in S_{(i, j)}$ there is $\theta_{q}(x, y) \in q$ which has a unique extension in $C_{(i, j)}$.

Proof Let $(a, b) \in D_{(i, j)}$ realize $q$. Then $\operatorname{tp}(a b / d)$ is isolated and, because $T$ is binary and $\varphi_{i}$ 's are $p$-principal, there is a formula $\theta_{q}(x, y) \in q(x, y)$ such that

$$
\varphi_{i}(d, x) \wedge \varphi_{j}(d, y) \wedge \theta_{q}(x, y) \vdash \operatorname{tp}(a b / d) .
$$

Since any extension of $\theta_{q}(x, y)$ in $C_{(i, j)}$ contains the formula on the left-hand side, we conclude that the extension is unique. 
Now, we claim that each $S_{(i, j)}$ is a discrete subset of $S_{p, p}(\varnothing)$. Suppose on the contrary that $q(x, y) \in S_{(i, j)}$ is an accumulation point of $S_{(i, j)}$. Then $\theta_{q}$ is contained in some $q^{\prime} \in S_{(i, j)}$ which is distinct from $q$. Thus $\theta_{q}$ has at least two extensions in $C_{(i, j)}$ : the one extending $q$ and the one extending $q^{\prime}$. This is a contradiction.

The first part of our theorem follows: if $n=1$, then $S_{(1,1)}=S_{p, p}(\emptyset)$ is discrete and, because it is compact, it has to be finite. Then by Corollary 2.4, $T$ has the strict order property. This is a contradiction. Therefore $n \geq 2$.

The second part follows from the following topological fact: a compact space which is a union of $m$ discrete subsets has CB-rank smaller than $m$ (easily proved by induction). In our situation $S_{p, p}(\emptyset)=\bigcup_{(i, j)} S_{(i, j)}$ is a union of $n^{2}$ discrete subsets, so $\mathrm{CB}\left(S_{p, p}(\emptyset)\right)<n^{2}$.

\section{References}

[1] Baizhanov, B. S., S. V. Sudoplatov, and V. V. Verbovskiy, "Conditions for non-symmetric relations of semi-isolation," Siberian Electronic Mathematical Reports, vol. 9 (2012), pp. 161-84. MR 2954703. 555, 558, 563

[2] Benda, M., "Remarks on countable models," Fundamenta Mathematicae, vol. 81 (1973/74), pp. 107-19. Zbl 0289.02038. MR 0371634. 561, 565

[3] Ikeda, K., A. Pillay, and A. Tsuboi, "On theories having three countable models," Mathematical Logic Quarterly, vol. 44 (1998), pp. 161-66. Zbl 0897.03035. MR 1622310. DOI 10.1002/malq.19980440203. 556, 563

[4] Peretjat'kin, M. G., "Complete theories with a finite number of countable models" (in Russian), Algebra i Logika, vol. 12 (1973), pp. 550-76; English translation in Algebra and Logic, vol. 12 (1973), pp. 310-26. Zbl 0298.02047. MR 0354347. 555, 570

[5] Pillay, A., "Instability and theories with few models," Proceedings of the American Mathematical Society, vol. 80 (1980), pp. 461-68. Zbl 0455.03012. MR 0581006. DOI 10.2307/2043741. 556, 558, 563, 565

[6] Saffe, J., E. A. Palyutin, and S. S. Starchenko, "Models of superstable Horn theories" (in Russian), Algebra i Logika, vol. 24 (1985), pp. 278-326; English translation in Algebra and Logic, vol. 24 (1985), pp. 171-210. Zbl 0597.03017. MR 0832910. 561

[7] Sudoplatov, S. V., "Powerful types in small theories," Siberian Mathematical Journal, vol. 31 (1990), pp. 629-38. Zbl 0723.03018. MR 1083739. DOI 10.1007/BF00970635. 560

[8] Sudoplatov, S. V., The Lachlan Problem (in Russian), Edition of Novosibirsk State Technical University, Novosibirsk, Russia, 2009. 555, 560, 570

[9] Tanović, P., "Theories with constants and three countable models," Archive for Mathematical Logic, vol. 46 (2007), pp. 517-27. Zbl 1115.03026. MR 2321591. DOI 10.1007/s00153-007-0054-2. 563

[10] Tanović, P., "Asymmetric RK-minimal types," Archive for Mathematical Logic, vol. 49 (2010), pp. 367-77. Zbl 1192.03005. MR 2609988. DOI 10.1007/s00153-010-0176-9. 560,563

[11] Vaught, R. L., "Denumerable models of complete theories," pp. 303-21 in Infinitistic Methods (Warsaw, 1959), Pergamon, Oxford, 1961. Zbl 0113.24302. MR 0186552. 555

[12] Woodrow, R. E., "A note on countable complete theories having three isomorphism types of countable models," Journal of Symbolic Logic, vol. 41 (1976), pp. 672-80. Zbl 0373.02036. MR 0491135. 556

[13] Woodrow, R. E., "Theories with a finite number of countable models," Journal of Symbolic Logic, vol. 43 (1978), pp. 442-55. Zbl 0434.03022. MR 0491137. 555, 565 


\section{Acknowledgments}

The second author was supported by Ministry of Education, Science and Technological Development of Serbia grant ON 174026.

\section{Sudoplatov}

Sobolev Institute of Mathematics

Novosibirsk State Technical University

Novosibirsk State University

Novosibirsk, Russia

and

Institute of Mathematics and Mathematical Modeling

Almaty, Kazakhstan

sudoplat@math.nsc.ru

http://math.nsc.ru/ sudoplatov

Tanović

Mathematical Institute of the Serbian Academy of Sciences and Arts

Faculty of Mathematics

University of Belgrade

Belgrade, Serbia

tane@mi.sanu.ac.rs 